\title{
ARFID: old wine in a new bottle?
}

Glenn Waller (1)

\section{COMMENTARY ON... AVOIDANT/RESTRICTIVE FOOD INTAKE DISORDER ${ }^{\dagger}$}

\begin{abstract}
SUMMARY
In this commentary, some aspects of the context of this diagnosis are considered. It is suggested that avoidant/restrictive food intake disorder (ARFID) is principally a reconfiguration of existing problems. However, it is also stressed that we still need to develop treatments that help patients and their families, given the expectation that has been fuelled by the promotion of the new diagnostic scheme. In developing those therapies, we should not forget that we previously had a number of psychosocial treatment approaches that were helpful for such children when their problems were labelled differently.
\end{abstract}

\section{DECLARATION OF INTEREST}

None.

\section{KEYWORDS}

ARFID; eating and feeding disorders; treatment; diagnosis.

Coglan \& Otasowie (2019, this issue) address a hot topic in the field of eating disorders - how do we understand eating problems in younger people when those problems are not anorexia nervosa? Their article has many educational benefits for clinicians. In particular, the guides to diagnosing an avoidant/restrictive food intake disorder (ARFID) and differentiating it from anorexia nervosa are helpful, and would be well placed on the clinician's wall, to remind them what to ask when such a case presents to them. However, there are gaps too

The article would have benefited from more of a history of such cases, to put this diagnostic development into context - after all, we can learn a lot from what went wrong in the past. Such cases have been known for many years, under a range of labels selective eating, food refusal, food phobia, food avoidance emotional disorder, etc. (e.g. BryantWaugh 2010). However, the use of such classifications was relatively rare under DSM-IV (American Psychiatric Association 1994), and it was very common to find that clinicians had never heard of these labels. In short, if the diagnosis was not anorexia nervosa, few clinicians knew what to do when it came to diagnosing, managing and treating such a case. Surprisingly, few clinicians working in adult eating disorder services realised that these foodrelated behavioural patterns are found in adulthood too, leaving the clinicians at a loss. Of course, the lack of awareness meant that many children and adults were not appropriately diagnosed. Given the young age of most individuals with such problems, many families experienced substantial and extended distress and frustration as a result of this gap in the field.

\section{Reconfiguration of existing knowledge}

Therefore, the DSM-5 diagnosis of ARFID (American Psychiatric Association 2013) needs to be treated more as a reconfiguration of existing knowledge than as reflecting a new entity. The main effect of the ARFID diagnosis is to pull three known behavioural patterns into a single diagnostic framework:

- lack of interest in food (e.g. the child who reports a chronic lack of appetite)

- limited range of sensory preferences (e.g. the child who selectively eats only white foods)

- fearful responses to food (e.g. not eating for fear of choking).

When the old labels (such as selective eating, food refusal, food phobia, food avoidance emotional disorder) were predominant, each of these three behavioural patterns had been treated successfully using largely behavioural, exposure-based methods (e.g. Nicholls 2001). However, such methods were only applied when the disorders were recognised, which was far less often than should have been the case.

So, what prevented the DSM-IV and other categorisations from capturing the public imagination? Could it be as simple as not having a banner term, such as ARFID? Quite possibly. It is also worth noting that two strongly interlinked developments appear to be key to the higher profile of ARFID:

- greater levels of advocacy via parent/carer organisations

- social media as a mechanism for raising awareness of ARFID.
Glenn Waller is Professor of Clinical Psychology at the University of Sheffield. He has worked clinically and researched in the field of eating disorders for over 35 years. He is former President of the international Academy for Eating Disorders. He served on the NICE (2017) Eating Disorders Guideline Development Group.

Correspondence Glenn Waller, Department of Psychology, University of Sheffield, Cathedral Court, 1 Vicar Lane, Sheffield S1 2LT, UK. Email: g.waller@sheffield.ac.uk

First received 4 Nov 2018 Final revision 8 Dec 2018 Accepted 9 Dec 2018

\section{Copyright and usage} (c) The Royal College of Psychiatrists 2019

${ }^{\dagger}$ See this issue. 
In short, we should see the emergence of ARFID in DSM-5 as a welcome opportunity to 'relaunch' these eating and feeding disorders of childhood, only this time with a greater chance of supporting families in seeking help (and often in educating clinicians about the disorders and how to help families). However, there is a rather sharp twist in the tail what 'help', exactly? As Coglan \& Otasowie point out, this condition is not included in established guidelines, meaning that we have a label for these behaviours but we are still in the early stages of developing treatments to offer the sufferers and their families (e.g. Thomas 2018a).

\section{The benefit of a label}

In explaining their development of a new form of cognitive-behavioural therapy (CBT) for ARFID, Thomas \& Eddy have pointed out that successfully promoting the construct of ARFID has definitely raised awareness (Thomas 2018b). Particularly among parents and carers of children and adolescents, the label of 'ARFID' meant that many realised that their child had a 'proper' problem that they could talk about with their family doctor, and the doctor had a label to use in order to make a referral onward. Previously, many of these parents would have been told that their child's eating problem was not anorexia nervosa, so that was that - no help would be known about or forthcoming. Now, DSM-5 (and, presumably, the forthcoming ICD-11, which usually follows suit) is telling families and clinicians that these children have a real problem, so they need help. That is where the problem emerges - what help? ARFID only emerged as a construct in 2013, and without any evidence about what to do to help. Consequently, as Thomas $\&$ Eddy (2018b) point out and as is clear from Coglan $\mathcal{E}$ Otasowie's article, we are only just beginning to produce evidence about ARFID's pathology and evidence-based treatments. Therefore, clinicians are receiving a lot of requests for help, but do not have much evidence on what to do for these patients and their families. In short, we are still developing the treatments as we go along, and all the while there are understandably frustrated families who expect us to know what to do to help their child recover.

\section{We know what to call it, but how do we treat it?}

In many ways, Coglan $\&$ Otasowie's article shows that it is the treatment recommendations that are the weakest element of this area. The guidance they give under all headings is a combination of general clinical management (which would have been better as a series of simpler bullet points) and speculation about psychological therapies that are in development (which would have been better in about 3 years, when current innovations in CBT, family-based treatment and other interventions are more developed). Authors such as Fitzpatrick, Lock, Bryant-Waugh and Thomas \& Eddy offer us substantial hope for such therapies, and their work on treatment for ARFID deserves our attention over the coming years. However, we are not there yet, making this article up to date but frustratingly incomplete (through no fault of the authors). Having said that, there is a surprising omission from the literature covered - what about the behavioural, exposure-based interventions that were useful for these behaviours before we labelled them differently? Changing the name might have been useful in promoting awareness of these cases, but that change does not mean that we have to forget the treatments that we used previously (e.g. Nicholls 2001). When we bear that in mind, it becomes less supportable for Coglan $\&$ Otasowie to conclude that we should consider using 'chronic disease management models'.

So, is this simply an instance of old wine in new bottles? The wine has not changed essentially - we always knew about these disorders. More positively, relaunching the wine in the new ARFID bottle has been successful in getting such cases identified. Frustratingly, we are still looking for the best corkscrew to open the bottle - those therapies are still in development. However, we should not forget the possibility that those old behavioural methods are effective for this new diagnosis.

\section{References}

American Psychiatric Association (1994) Diagnostic and Statistical Manual of Mental Disorders (4th edn) (DSM-IV). APA

American Psychiatric Association (2013) Diagnostic and Statistical Manual of Mental Disorders (5th edn) (DSM-5). American Psychiatric Publishing.

Bryant-Waugh R, Markham L, Kreipe RE, et al (2010) Feeding and eating disorders in childhood. International Journal of Eating Disorders, 43: 98-111.

Coglan L, Otasowie J (2019) Avoidant/restrictive food intake disorder: what do we know so far? BJPsych Advances, 25: 90-8.

Nicholls D, Christie D, Randall L, et al (2001) Selective eating: symptom, disorder or normal variant. Clinical Child Psychology and Psychiatry, 6: 257-70

Thomas JJ, Wons OB, Eddy KT (2018a) Cognitive-behavioral treatment of avoidant/restrictive food intake disorder. Current Opinions in Psychiatry, 31: $425-30$.

Thomas JJ, Eddy KT (2018b) Cognitive-Behavioral Therapy for Avoidant/ Restrictive Food Intake Disorder (CBT-AR): Children, Adolescents, and Adults. Cambridge University Press. 